a great deal of thought. Though it is designed for use in a children's hospital, I think that, ideally, when further improvements have been made, the finally agreed changes should be incorporated in a revised World Health Organisation manual. The concept of a uniform international classification of disease is essential to the orderly advance in scientific knowledge made possible by statistical analysis on a large scale by computer.

Virus Diseases and the Nervous System. A Symposium edited by C. W. M. WhItTY, J. T. Hughes, and F. O. MacCallum. (Pp. xii +259 ; 61 figures. 65s.) Oxford and Edinburgh: Blackwell Scientific Publications. 1969.

Based on a symposium held in Oxford in July 1968, this book has three main sections-on acute virus diseases of the nervous system, slow virus diseases, and associations between viruses and malignancy. The most remarkable recent advances have concerned twó rare human diseases and one of sheep. Kuru is confined to New Guinea, transmitted by cannibalism, and caused by an agent with unusual properties. Subacute sclerosing leucoencephalitis is now known to be due to 'temperate' infection by measles virus. Scrapie is a recessively inherited disease of sheep, but a transmissible factor ('provirus') is produced in the affected animal. It remains to be seen whether these discoveries will prove relevant to commoner problems like disseminated sclerosis or will remain scientifically fascinating oddities. More conventional aspects of CNS virology are generally well covered, though naturally a symposium is not a textbook; it is interesting that poliomyelitis can now go virtually unmentioned. The discussions are interesting and helpful, edited with a reasonable compromise between conciseness and colloquialism. This is a difficult task, and in places the editorial pencil could have been used more drastically. Rather than solemnly print 'a paper in the Journal of Experimental Medicine, I'm afraid I forget the authors', it would have been better to ask the discussant to supply the exact reference. It is also unsatisfactory to pass a sentence which implies, even if unintentionally, that chickenpox is caused by herpes simplex virus (p. 69). However, the editors are to be congratulated on having this book published within a year of the symposium. The paediatrician will find something of clinical value in it, and a good deal more of general medical and scientific interest.

\section{Some Inherited Disorders of Brain and Muscle.}

Proceedings of The Fifth Symposium of The Society for the Study of Inborn Errors of Metabolism. Edited by J. D. AllaN and D. N. RaINe. (Pp. viii + 154; illustrated. 40s.). Edinburgh and London: E. \& S. Livingstone Ltd. 1969.

There is no doubt that symposia and publication of their proceedings serve a useful purpose in any branch of knowledge which is rapidly advancing. The two main themes of this book are concerned with diseases of muscle and with disorders of sphingolipid meta- bolism. After a review of the clinical features of muscular dystrophy, the biochemical abnormalities are considered and interesting speculations are made on the possible role of the nervous system in muscular dystrophy. The forms of glycogen storage disease involving muscle are also reviewed. The various types of sphingolipidoses are considered from both the biochemical and neuropathological point of view and there are also papers on Wilson's disease and on genetic aspects of metabolic disorders. To the patient suffering from muscular dystrophy the progress of research into possible causes must seem painfully slow, but a number of recent advances in the understanding of the chemical changes in dystrophic muscles are well reviewed. However, the ways in which disorders of glycogen metabolism can interfere with muscle function are perhaps of greater fascination at the moment, even if such conditions are much rarer. The classification of the cerebral lipidoses in chemical terms is of especial interest, but there is obviously much still to be learnt. The subject is becoming clearer, though it may still be confusing to those with little biochemical training.

This book can undoubtedly be recommended to those interested in these subjects, who wish to keep abreast of advances in research. The symposium does seem to have fulfilled its purpose, as defined by one of the contributors; to provoke thought and discussion.

Disorders of Voluntary Muscle, 2nd edition. Edited by John N. Walton. (Pp. $941 ; 198$ figures. 200s.) London: J. and A. Churchill. 1969.

The second edition of this book is half as long again and costs twice as much as the original, published 5 years ago. Its many merits were referred to in the previous review in the Archives (40, 234, 1965). It will certainly remain the standard and indispensable reference work on muscle disorders. Some of the increase in length has been made necessary by the rapid advances in techniques for studying muscle, which are well described. However, the multiple authorship does seem to have resulted in more repetition than is really needed by the editor's desire that each chapter should stand as a comprehensive essay in its own right. The pathology of nemaline myopathy is described in similar terms, though at varying length, in 5 different places. Here, as elsewhere, some economy could surely have been made by cross-references. It would be useful instead to hear a little more about the clinical picture of this condition, and it would be tidier if its original description were not attributed to two different sets of authors on p. 162 and p. 303.

It is too much to hope that the next edition will cost less, but at least it should be made more compact. This book is much too useful to be allowed to suffer a pseudo-hypertrophic dystrophy.

Atlas of Mental Retardation Syndromes: Visual Diagnosis of Facies and Physical Findings. Edited by DrS. SIDNEY S. GeLlis and MURRAY FEINGOLD. (Pp. $\mathrm{x}+188$; illustrated with colour 